\title{
Blood glucose monitors: a laboratory and patient assessment
}

\author{
DAVID J WEBB, J M LOVESAY, A ELLIS, A H KNIGHT
}

\section{Summary and conclusions}

The four blood glucose monitors available in the United Kingdom were compared by asking the opinions of 24 patients who used each monitor for two weeks, by correlating their blood glucose results with those obtained in the laboratory, and by having the monitors examined by an electronics engineer. Of the two batteryoperated monitors, patients preferred the Hypocount (15) to the Glucochek (9). The mains-operated units were less popular, with little to choose between Eyetone and Reflomat. Under field conditions the blood glucose results obtained with the Glucochek correlated poorly with the standard reference method. In contrast the Hypocount, Eyetone, and Reflomat machines produced good correlations. Poor results with the Glucochek were mainly due to faulty timing systems.

The patients' preference for the Hypocount was supported by tests of performance under laboratory conditions and by the electronics engineer's report.

\section{Introduction}

Self-monitoring of blood glucose levels is becoming an accepted and valuable advance in the management of diabetes. ${ }^{1-4}$ Although not suitable for every insulin-dependent patient, the technique offers the possibility of better and safer control of diabetes, shows the limitations of urine tests, and for the first time gives diabetics the information they need to take an active part in controlling their condition. Although the patient has to obtain a drop of capillary blood by needle or lancet, most patients prefer it to urine testing. ${ }^{1}$

Some patients have already bought their own monitors and others are being encouraged to do so. But, with four products available in the United Kingdom, their problem is in deciding which one to buy. In attempting to pick a "best buy" we assessed the four available monitors in three ways: by analysing the opinions of 24 patients, by comparing the performance of the units under field and laboratory conditions, and by seeking the opinion of an electronics engineer.

\section{Patients and methods}

The 24 insulin-dependent patients who tested the monitors comprised 12 men and 12 women aged 16 to 60 years (mean 35) who had had diabetes for 3 months to 35 years (mean 10). One patient had had experience of self-monitoring with an Eyetone machine and one with a Glucochek. One patient was partially sighted but could test urine with Clinitest tablets and administer insulin with a Click-count syringe. Our own experience consisted of five years of using the Eyetone and a few weeks with Glucochek. Initially 24 new monitors were assessed. Six Eyetone, six Hypocount, and six Reflomat machines were kindly lent by their manufacturers. Our clinic had recently

\footnotetext{
Medical Day Unit, Department of Medicine, Stoke Mandeville Hospital, Aylesbury, Buckinghamshire

DAVID J WEBB, MB, BS, senior house officer

J M LOVESAY, SRN, sister-in-charge

A ELLIS, BA, biochemist

A H KNIGHT, MB, MRCP, consultant physician
}

bought six Glucochek machines, but they had not been used. (During the trial period two further Glucochek monitors were included.)

With all of the machines the patient places a drop of blood on an enzyme-impregnated reagent strip (Dextrostix or Reflotest) and removes it after one minute. The colour change, dependent on the glucose content of the blood, is determined by the reflectance of light in the previously calibrated monitor.

After a seven-day practice period each patient used each type of monitor for two weeks. Patients performed at least two tests a day, and they were encouraged to take the monitors with them to work or while travelling. Each week they attended our unit, where a venous blood sample was taken by a doctor or nurse. We then observed the patients while they tested part of the sample with the monitor they were assessing. The rest of the sample was analysed by Technicon autoanalyser in our biochemistry department. The patient's technique was thus checked and correlations of blood glucose measurement between each monitor type and the autoanalyser were made on the basis of two correlations per monitor per patient. The patients were not told the laboratory results. At the end of each fortnight the patient changed to a different type of monitor. At the end of the eight weeks each patient completed a detailed questionnaire and assessed various features of each machine on a four-star scale. All 26 monitors were then tested in the laboratory. The built-in timers on the Glucochek and Hypocount monitors were checked against a stopwatch. Tests of linearity were made using tech-chek (a set of calibration solutions of known values $2 \cdot 5,5 \cdot 0,7 \cdot 2$, and $13.9 \mathrm{mmol} / \mathrm{l}$ supplied by Ames) or with freshly drawn blood samples. Reproducibility tests were made with fresh blood samples of known glucose content tested at least five times on each monitor.

One of each of the four types of monitor was examined by our area health authority electronics engineer. He assessed their conformity with DHSS requirements where applicable (Hospital Technical Memorandum No 8), but did no electronic or durability tests. Table I shows the basic data on the four monitors.

TABLE I-Basic data on the four monitors

\begin{tabular}{|c|c|c|c|c|}
\hline & Eyetone & Reflomat & Glucochek & Hypocount \\
\hline Manufacturer & $\begin{array}{l}\text { Ames } \\
\text { Company }\end{array}$ & $\begin{array}{l}\text { Boehringer } \\
\text { Corporation }\end{array}$ & $\begin{array}{l}\text { Medistron } \\
\text { Ltd }\end{array}$ & $\begin{array}{l}\text { Hypoguard } \\
\text { Ltd }\end{array}$ \\
\hline $\begin{array}{l}\text { Warm up time } \\
(\min )\end{array}$ & 30 & 5 & 0 & 0 \\
\hline $\begin{array}{l}\text { Timer incorpo- } \\
\text { rated }\end{array}$ & & & Yes & Yes \\
\hline Calibration & $\begin{array}{l}\text { 2-point refer- } \\
\text { ence strips }\end{array}$ & $\begin{array}{l}\text { 2-point refer- } \\
\text { ence system }\end{array}$ & $\begin{array}{l}\text { Zero calibration } \\
\text { reference } \\
\text { strip }\end{array}$ & $\begin{array}{l}\text { From unused } \\
\text { test strip }\end{array}$ \\
\hline $\begin{array}{l}\text { Range of scale } \\
\text { (mmol/1) } \\
\text { Stated accuracy }\end{array}$ & $\begin{array}{l}0.5-22 \cdot 0 \\
0.28 \mathrm{mmol} / 1 \\
\quad \text { (in range } \\
0.5-14 \text { ); } \\
0.55 \mathrm{mmol} 1 \\
\text { (in range } \\
14-22)\end{array}$ & $\begin{array}{l}0.55-20 \cdot 0 \\
\pm 10 \%\end{array}$ & $\begin{array}{l}0.0-25 \cdot 0 \\
\text { greater of } \\
\quad 15 \% \text { or } 0.5 \\
\mathrm{mmol} / 1\end{array}$ & $\begin{array}{l}0 \cdot 0-24 \cdot 0 \\
\pm 10 \%\end{array}$ \\
\hline $\begin{array}{l}\text { Data display } \\
\text { Cost to private } \\
\text { purchaser } \\
\text { (exempt from } \\
\text { VAT) }\end{array}$ & Needle scale & Calibrated dial & Digital & Digital \\
\hline
\end{tabular}

Cost of Dextrostix (excluding VAT): $£ 2.30$ per 10 strips (foil-wrapped), $£ 2.65$ pe 25 strips (bottled); cost of Reflostrips (excluding VAT) $\$ 2.55$ per 25 strips. (All prices through hospital pharmacy.)

\section{Results}

CONSUMER OPINION

Table II shows the patients' opinions on various features of the four monitors. Digital display of results was popular, and the larger digits of the Hypocount scored the highest rating. The partially sighted patient found the Hypocount the easiest to read and the Reflomat the hardest. 
TABLE II-Patients' opinions on aspects of the four monitors. Star ratiings (four stars $=$ best) are means of 24 opinions

\begin{tabular}{lcccc}
\hline & Eyetone & Reflomat & Glucochek & Hypocount \\
\hline $\begin{array}{l}\text { Comprehensiveness of } \\
\text { printed instructions }\end{array}$ & $* * * *$ & $* * *$ & $* * *$ & $* *$ \\
$\begin{array}{l}\text { Clarity of printed } \\
\text { instructions }\end{array}$ & $* *$ & $* * *$ & $* * * *$ & $* * * *$ \\
$\begin{array}{l}\text { Ease of calibration } \\
\text { Confidence in accuracy }\end{array}$ & $*$ & $* *$ & $* * * *$ & $* * *$ \\
$\begin{array}{l}\text { Display of blood glucose } \\
\text { result }\end{array}$ & $* *$ & $*$ & $* * *$ & $* * * *$ \\
$\begin{array}{l}\text { Overall presentation of } \\
\text { monitor }\end{array}$ & $*$ & $* *$ & $* * * *$ & $* * *$ \\
$\begin{array}{l}\text { Portability and convenience } \\
\text { No of tests needed to } \\
\text { become competent }\end{array}$ & $*$ & $*$ & $* * * *$ & $* * *$ \\
$\begin{array}{l}\text { Time taken to calibrate and } \\
\text { perform test once }\end{array}$ & $2(1-5)$ & $3(1-12)$ & $1 \cdot 5(1-3)$ & $1 \cdot 7(1-4)$ \\
$\quad$ competent (min) & $3 \cdot 2(2-5)$ & $3 \cdot 6(2-7)$ & $2 \cdot 1(1 \cdot 5-3)$ & $2 \cdot 1(2-3)$ \\
$\begin{array}{l}\text { Nof monitor faults } \\
\text { detected by patients }\end{array}$ & 0 & 1 & 3 & 0 \\
\hline
\end{tabular}

None of the Eyetone or Hypocount monitors gave any trouble, one Reflomat broke down three days before the end of the study owing to a faulty component which required correction, and the Glucochek monitors developed three faults. One Glucochek monitor failed completely and was returned to Medistron, who replaced the battery and faulty components, while the other two were reported to have inaccurate timers. This was noted almost at the end of the trial and these monitors were therefore kept in use but the patients advised not to use the built-in timer.

Several patients reported problems in recharging the Glucochek's battery. Often the low-battery-state warning light suggested that two overnight recharges per week were insufficient, and on one monitor the light appeared to glow at random. In one case the machine apparently failed to recharge, but the jack plugs from the recharging units fitted very loosely with all the Glucochek monitors.

All but one patient preferred battery power; 21 regarded the facility to calibrate the monitor before each test as important; six preferred the Glucochek timing system, six the Hypocount, and 12 had no preference; nine patients preferred the Reflotest strip system (used in the Reflomat) while eight preferred Dextrostix (used in the other three monitors) and seven stated no preference. Table III shows the patients' overall preferences.

TABLE III-Patients' preferences for the monitor they would prefer to own (a) if they had to buy it themselves (left-hand columns) and (b) taking all other factors into account but disregarding cost (right-hand columns)

\begin{tabular}{lrrrrrcrcr}
\hline & \multirow{2}{*}{$\begin{array}{c}\text { Eyetone } \\
\text { overall }\end{array}$} & \multicolumn{2}{c}{$\begin{array}{c}\text { Reflomat } \\
\text { overall }\end{array}$} & $£$ & $\begin{array}{c}\text { Glucochek } \\
\text { overall }\end{array}$ & $£$ & $\begin{array}{c}\text { Hypocount } \\
\text { overall }\end{array}$ \\
\hline 1st & 0 & 0 & 0 & 1 & 9 & 9 & 15 & 14 \\
2nd & 3 & 3 & 3 & 4 & 9 & 8 & 9 & 9 \\
3rd & 9 & 8 & 12 & 11 & 2 & 4 & 0 & 1 \\
4th & 12 & 13 & 9 & 8 & 4 & 3 & 0 & 0
\end{tabular}

\section{LABORATORY RESULTS}

The figure shows the correlation between blood glucose values obtained with the four monitors and those from the autoanalyser. The autoanalyser measures total reducing substances in the blood and should therefore produce a slightly higher value than the Dextrostix and Reflotest systems, which measure glucose alone. Correlation was good for all Eyetone, Hypocount, and Reflomat monitors. Four Glucochek monitors correlated well, one correlated poorly, two seemed to correlate poorly but the quantity of data was limited, and for one there was insufficient data.

The built-in timers on the six Hypocount monitors consistently timed 62 seconds. Most of the Glucochek monitors had faulty timers: after a full overnight charge only one consistently timed a 60 -second minute, although two others became consistent after a few minutes' warm-up. All four types of monitor yielded acceptably accurate results in terms of linearity and showed good reproducibility (coefficients of variation: Eyetone $6.24 \%$, Glucochek $10.63 \%$, Hypocount $7 \cdot 60 \%$, Reflomat $4.35 \%$ ).

\section{ELECTRONICS REPORT}

Of the two mains-operated monitors the engineer preferred the Eyetone to the Reflomat since it was likely to be more reliable in the long-term. The Eyetone conformed to HTM8 standards, but the Reflomat would need minor modifications. He preferred the Hypocount to the Glucochek because it had better mounted batteries and no preset potentiometers that could cause problems with age.

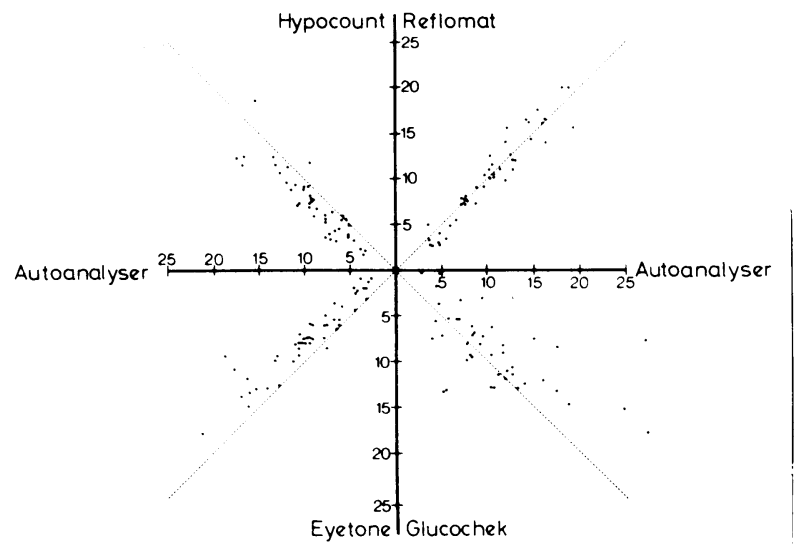

Correlations between results from glucose monitors and those from the autoanalyser. Values are mmol glucose per litre. Conversion: SI to traditional units-Glucose: $1 \mathrm{mmol} / 1 \approx$ $18 \mathrm{mg} / 100 \mathrm{ml}$.

\section{Discussion}

It was no surprise that our patients favoured the two (more portable) battery-operated models. Simple addition of the star ratings in table II would not at first suggest that more preferred the Hypocount, but these features were offset by the number of operational faults and the fact that the Glucochek seemed to need recharging more often.

The laboratory assessment of accuracy leads us to support the majority choice of our patients. The Hypocount was operationally trouble-free and its results correlated well with those of a standard method. On grounds of design the engineer also preferred the Hypocount. The Hypocount timers were consistent at 62 seconds. Although two seconds longer than recommended for the Dextrostix reaction time, this did not seem to have any practical importance since the linearity and reproducibility results suggested that the manufacturer's calibration had taken this into account. On the other hand, many of the Glucochek monitors had erratic timers, which were probably responsible for their poorer accuracy. Excluding the timer brought the results under laboratory conditions just within an acceptable range. A coefficient of variation of less than $10 \%$ is acceptabic since much of the overall error of any monitor will be due to the nature of the test strips themselves.

We understand from Medistron Ltd that it has now modified the Glucochek timer so that the timer does not have to be warmed up before producing a consistent timing of 60 seconds. All new Glucochek monitors have been modified, and since 20 September Medistron have recommended that this modification be carried out on all the earlier units sold (up to serial number 2100). Medistron also said that some early monitors had too high a setting for the low-battery-state warning light.

Although the calibration of the two battery-operated models is preset by the manufacturer, we and most of our patients think that some form of calibration check across the range should be available to the patient, who will otherwise have no means of recognising a malfunction unless it be gross.

Between the two mains-operated monitors, the division of patient opinion was much closer, with the Reflomat marginally more popular. The Reflomat performed with the best reproducibility. The present Reflotest glucose system does not read accurately below $3.0 \mathrm{mmol} / 1$ but a modification of the test strip (Reflotest Hypoglycemie) is available to cover the range 0.55 to $8.3 \mathrm{mmol} / \mathrm{l}$. When cost was not being considered one patient gave Reflomat as overall first choice. The greater difficulty in 
calibrating the Eyetone was perhaps a significant factor in our patient's choice. For hospital use a mains-operated monitor is usually desirable. Our electronics engineer gave marginal preference for Eyetone. Reflomat, however, has a potential advantage in that the Reflotest system holds its colour and reading, which could be an advantage under certain circumstances. ${ }^{4}$ Both the Eyetone and the Reflomat gave good results. Our experience with Eyetone monitors over several years has been favourable and the after-sales service excellent.

For self-monitoring, however, further advances in technology are expected. The BM-Test-Glycemie $20-800$ strip (Boehringer) now available is yielding encouraging results and may provide a system of monitoring without the need for an actual monitor. Nevertheless, of the four monitors currently available our triple assessment of patient preference, laboratory performance, and electronic opinion gave first place to Hypocount.
We acknowledge the help and encouragement of the British Diabetic Association and the helpful co-operation of the Ames, Boehringer, Hypoguard, and Medistron companies.

\section{References}

${ }^{1}$ Sönksen PH. Home-monitoring of blood glucose. British Diabetic Association-Clinic Newsletter. June 1979:4-7.

2 Sönksen PH, Judd SL, Lowy C. Home monitoring of blood-glucosemethod of improving diabetic control. Lancet 1978; :729-32.

${ }^{3}$ Walford S, Allison SP, Gale EAM, Tattersall RB. Self-monitoring of blood-glucose-improvement of diabetic control. Lancet 1978; ; : 732-5.

${ }^{4}$ Howe-Davies S, Holman RR, Phillips M, Turner RC. Home blood sampling for plasma glucose assay in control of diabetes. $\mathrm{Br} \mathrm{Med} \mathcal{F}$ 1978;ii:596-8.

(Accepted 15 November 1979)

\title{
Protective effect of inhaled salbutamol powder in children assessed by histamine challenge
}

\author{
C R PULLAN, A J MARTIN
}

\section{Summary and conclusions}

A double-blind placebo-controlled trial of 100,400 , and $800 \mu \mathrm{g}$ inhaled salbutamol powder was conducted on 12 children. The protective effect at 10 minutes, two hours, and four hours was assessed by histamine challenge. At 10 minutes there was good protection with all doses, but by four hours there was significant protection only with $800 \mu \mathrm{g}(\mathbf{p}<0.01)$. Salbutamol powder may need to be taken at least every four hours for complete protection. There was a dose-related effect with a single dose of up to $800 \mu \mathrm{g}$; increasing the dose increased the effect and duration of action.

Currently advertised dose regimens of salbutamol powder for children (200 $\mu$ g three or four times a day) are apparently submaximal. Histamine challenge is a satisfactory method of assessing the protective effect of a drug in asthmatic children.

\section{Introduction}

Inhaled salbutamol is well established as a valuable treatment of wheezing in children. ${ }^{1}$ Many young children have difficulty mastering the metered aerosol ${ }^{2}$ but can use a dry powder inhaler. ${ }^{3}$ Young children unable to use a metered aerosol who require inhaled salbutamol have had to use air compressors to drive nebulisers, which are both cumbersome and expensive.

Salbutamol powder is now available for use in an inhaler (Rotahaler, Allen and Hanburys). This effectively increases the forced expiratory volume in one second $\left(\mathrm{FEV}_{1}\right)$ in adults ${ }^{4}$ and children. ${ }^{6}$ The recommended prophylactic dose is $400 \mu \mathrm{g}$ for adults and $200 \mu \mathrm{g}$ for children three or four times a day. This dose, however, may produce submaximal responses in

\footnotetext{
Department of Child Health, Royal Victoria Infirmary, Newcastle upon Tyne NE1 4LP

C R PULLAN, MB, MRCP, senior registrar

A J MARTIN, MB, MRCP, senior registrar
}

children, ${ }^{6}$ and Hetzel and Clark ${ }^{5}$ reported increasing effect in adults with cumulative doses of up to $800 \mu \mathrm{g}$. Many children with asthma have intermittent wheezing with a normal $\mathrm{FEV}_{1}$ for much of the time. ${ }^{7}$ Hence testing a single dose of a drug and its effect on $\mathrm{FEV}_{1}$ can be done only on a few more severely affected children. In the other cases, however, episodes may still be frequent enough to warrant continuous treatment with salbutamol.

Histamine is a non-specific bronchoconstrictor in increased bronchial lability. ${ }^{8}{ }^{9}$ It is useful in assessing the protective effect of drugs, since the bronchoconstriction can be controlled and its effect is transient. ${ }^{10} \mathrm{We}$ decided to assess the protective effect of a single dose of salbutamol powder using inhaled histamine. The trial was approved by the local ethical committee.

\section{Subjects and methods}

Twelve children aged 6-13 years with episodic asthma were studied. All could perform satisfactory forced expiratory manoeuvres. Eleven had a baseline $\mathrm{FEV}_{1}$ of $70 \%$ or more of the predicted normal value on each day (normal values taken from Cogswell et $a l^{11}$ ). One boy had an $\mathrm{FEV}_{1}$ of $58 \%$ predicted on one day and between $70 \%$ and $90 \%$ on the other days. Only steroids were continued from 12 hours before the start of each study day until after the last histamine challenge of that day.

Histamine challenge-The histamine challenge procedure used was similar to that of Chai et al. ${ }^{12} \mathrm{FEV}_{1}$ was recorded on a Vitalograph. When consistent results were obtained the best of three was used as a baseline. Buffered histamine acid phosphate was used in roughly doubling concentrations from $0.03 \mathrm{mg} / \mathrm{ml}$ to $10 \mathrm{mg} / \mathrm{ml}$. Solutions were administered by facemask from a Bard Mini-Neb nebuliser driven by oxygen at $8 \mathrm{l} / \mathrm{min}$. Five inspiratory capacity breaths were taken first of diluent alone, and three minutes later the $\mathrm{FEV}_{1}$ was recorded. Provided there was no fall in $\mathrm{FEV}_{1}$ with the diluent this was repeated with increasing concentrations of histamine at fourminute intervals until the $\mathrm{FEV}_{1}$ had fallen by $20 \%$ or more. The test was then stopped. The concentration of histamine that would produce a $20 \%$ fall (provocation concentration $20 ; \mathrm{PC}_{20}$ ) was calculated from the dose-response curve. If the $\mathrm{FEV}_{1}$ did not fall by $20 \%$ with the top concentration $(10 \mathrm{mg} / \mathrm{ml})$ a $P_{20}$ was calculated by extrapolation up to $20 \mathrm{mg} / \mathrm{ml}$, but not beyond. 